\title{
Nivel de satisfacción laboral y Síndrome de Burnout en el profesional de enfermería en el Sistema de Atención Móvil de Urgencias.
}

Level labor satisfaction and burnout syndrome in the nursing professional of the emergency mobile attention system - Lima, 2017.

Verónica Gabriel Aranda ${ }^{1, \text { a }}$.

\section{RESUMEN}

Objetivo: Determinar la asociación entre satisfacción laboral y Síndrome de Burnout en el profesional de Enfermería que labora en el Sistema de Atención Móvil de Urgencias (SAMU). Material y métodos: estudio cuantitativo, correlacional, descriptivo y transversal. Se incluyó a la población de 70 profesionales de Enfermería, a quienes se les aplicó mediante la técnica de encuesta, el cuestionario de Satisfacción Laboral, el cual fue validado obteniendo alfa de Crombach $=0,76$ y el cuestionario Inventario de Burnout de Maslach (MBI) sin modificaciones. Resultados: Para la satisfacción laboral, se identificó que la dimensión de factores extrínsecos muestra el $40 \%$ de enfermeros con insatisfacción y el mismo porcentaje de enfermeros en la dimensión de factores intrínsecos se encuentran medianamente satisfechos. En el Síndrome de Burnout se encuentra que el 42,9\% de enfermeros presentan nivel alto en cuanto al agotamiento emocional y el 38,6\% nivel medio por ausencia de realización personal. Conclusiones: La mayoría de enfermeros se encuentran medianamente satisfechos en la dimensión de factores intrínsecos e insatisfechos en factores extrínsecos. Asimismo, enfermeros del presente estudio se encuentran con nivel alto de agotamiento emocional seguido de nivel bajo de despersonalización de Síndrome de Burnout. Sin embargo, no existe relación entre las variables.

PALABRAS CLAVE: Satisfacción en el trabajo, agotamiento profesional, enfermería.

\section{SUMMARY}

Objective: To determine the relationship between Job Satisfaction and Burnout Syndrome in the Nursing professional working in the Mobile Emergency Care System (SAMU) Lima - 2017. Material and methods: Quantitative, correlational, descriptive and cross-sectional study. The population was made up of 70 Nursing professionals. The technique that was used was the survey and as an instrument the Labor Satisfaction questionnaire, which was validated obtaining Crombach's alpha $=0.76$ and the Maslach Burnout Inventory (MBI) questionnaire without modifications. Results: Regarding job satisfaction, it was found that the extrinsic factor dimension shows $40 \%$ of nurses with dissatisfaction and the same percentage of nurses in the intrinsic factor dimension are moderately

Sistema de Atención Móvil de Urgencias, Ministerio de Salud. Lima, Perú

Enfermera Especialista en Centro Quirúrgico. 
satisfied. In Burnout Syndrome it is found that $42.9 \%$ of nurses have a high level of emotional exhaustion and $38.6 \%$ average level of lack of personal fulfillment. Conclusions: There is no relationship between the variables. Job satisfaction Most nurses are moderately satisfied in the dimension of intrinsic factors and dissatisfied in extrinsic factors. Likewise, nurses of the present study find themselves with a high level of emotional exhaustion followed by a low level of depersonalization of Burnout Syndrome.

KEYWORDS: Job satisfaction, burnout, psychological, nursing.

\section{INTRODUCCIÓN}

La satisfacción laboral es un objetivo importante dentro de una organización siendo ésta una respuesta positiva de la persona ante un puesto laboral, manteniendo el compromiso institucional y por lo tanto brindando una atención con calidad, como también son las actitudes que se demuestra en el trabajo; por el contrario, si hay insatisfacción laboral la persona podría presentar problemas en el trabajo, como por ejemplo el Síndrome de Burnout, entre otros, englobando a problemas biopsicosociales (1-3). Asimismo, respecto a la satisfacción laboral, se la identifica como la actitud que muestra el trabajador frente a su situación laboral, dicha actitud puede ser positiva o negativa por lo tanto, es importante considerar la satisfacción laboral como un componente crítico dentro de una institución. A ello se agrega que es un indicador de calidad que puede verse afectado por factores extrínsecos (Teoría Bifactorial de Herzberg) dentro del ámbito laboral (4). Según Menéndez la teoría de Herzberg supone la existencia de dos factores relevantes; el índice de satisfacción que permite reconocer si la persona ese encuentra más o menos satisfecha, aspecto que se puede medir por factores intrínsecos laborales y otro índice de la insatisfacción que permitiría identificar cuando la persona está más o menos insatisfecho, por lo que se puede valorar por los factores extrínsecos del trabajo (5).

Además, entendiendo la satisfacción laboral, como el nivel de felicidad que puede experimentar un individuo en su entorno laboral considerando sus expectativas personales coherentes con la realidad laboral (6); se podría comprender que esta satisfacción pueda repercutir en el desempeño laboral. Reflejo de ello puede ser que ante a disminución de esta, el empleado puede reducir la eficacia en su quehacer y al no estar motivado incluso podría abandonar la organización a la que pertenece en su entorno laboral. Por otro lado, un constante estrés en el ámbito laboral puede originar una respuesta negativa biopsicosocial llamado Síndrome de Burnout, que se caracteriza por percibir una sensación de fracaso y agotamiento. En muchos casos a causa del desgaste de energías y recursos. Esta situación puede desencadenar sensación de distanciamiento del trabajo, con la correspondiente baja satisfacción laboral y deterioro en la productividad (7). Otros autores mencionan que este síndrome afecta a los profesionales que interactúan con personas, siendo los profesores, médicos, enfermeros, los más afectados. Ésta variable de investigación es caracterizada por la despersonalización, cansancio emocional y baja realización personal, siendo éstas las dimensiones del instrumento más utilizado Maslach Burnout Inventory (8-11).

Por lo tanto, el Síndrome de Burnout en la actualidad se presenta como un problema de salud donde la persona se aísla de su entorno laboral presentando problemas de agresividad, irritabilidad, pérdida de energía, cansancio, entre otros. Podría llegar hasta perder sus ideales y hasta alejarse de su familia, entorno laboral y despreocupación de sí mismo (12).

El Síndrome de Burnout es valorado a través de un instrumento llamado Maslach Burnout Inventory (MBI), éste cuestionario es adecuado y adaptado al personal sanitario para el uso a nivel mundial. Dicho instrumento es evaluado mediante 3 dimensiones establecida por Maslach, siendo el agotamiento emocional, la despersonalización y la falta de realización personal (13).

La enfermería desarrolla actividades en el ámbito hospitalario y pre hospitalario como es el caso de los enfermeros que laboran en el Sistema de Atención Móvil de Urgencia (SAMU), un servicio de atención pre hospitalario que permite a todas las personas recibir asistencia médica de calidad y de manera oportuna ante una urgencia o emergencia.

La atención a cargo del profesional de Enfermería en el espacio pre hospitalario es brindar atención de salud en conjunto con el personal médico, realizar 
procedimientos invasivos o no invasivos, según necesidad y si es necesario el traslado asistido hacia un Establecimiento de Salud; por lo tanto, este personal tiene que estar preparado para las diferentes situaciones externas (bulla, desesperación del entorno familiar, espacios angostos o estrechos para la atención, factor climático, etc.) para el desarrollo adecuado de las actividades.

La satisfacción laboral y el Síndrome de burnout son variables que afectan al desarrollo profesional, emocional de los profesionales de la salud, ambos estudiados en las diferentes especialidades del área asistencial, también existen estudios en el área de educación, empresas privadas, entre otros. Con el estudio de ambas variables se logra medir el grado de compromiso institucional, calidad de atención a los pacientes en atenciones de urgencia y emergencia. Asimismo éste profesional podrá afrontar los cambios en la institución, asumiendo la responsabilidad y el compromiso para el desarrollo en el cumplimiento de los objetivos y la atención adecuada a los pacientes (14).

El SAMU en el Perú fue creado con la finalidad de brindar atención de urgencia y emergencia en las diferentes situaciones del ámbito pre hospitalario, atención realizada por el profesional médico $\mathrm{y}$ enfermero. Teniendo menos de una década de desarrollo no se evidencia mayores estudios de investigación en dicho personal y por lo tanto no se conoce la prevalencia de Síndrome de Burnout y la Satisfacción Laboral en este sector.

El objetivo del presente trabajo fue identificar si existe relación entre la satisfacción laboral y el nivel del síndrome de burnout en el profesional de Enfermería que labora en el Sistema de Atención Móvil de Urgencias de Lima.

\section{MATERIAL Y MÉTODOS}

Es un estudio correlacional, cuantitativo, descriptivo de corte transversal. La población de estudio estuvo conformada por 80 profesionales de Enfermería que laboran en el Sistema de Atención Móvil de Urgencias. Sin embargo, solo 70 enfermeros cumplieron los criterios de inclusión de ser nombrados o contrato CAS y que aceptaron participar en el estudio de forma anónima y voluntaria.

Para recabar la información necesaria se utilizaron dos instrumentos empleando en ambos la Escala
Likert. Se realizó la creación del instrumento de Satisfacción Laboral, y se validó por juicio de expertos con prueba binomial, y para la confiabilidad consideró prueba piloto, obteniendo un alfa de Crombach $=0,76$. Para medir el Síndrome de Burnout se empleó el cuestionario de MBI (Inventario de Burnout de Maslach) sin realizar modificaciones (15).

Los datos obtenidos de los cuestionarios aplicados a los profesionales de Enfermería fueron procesados a través del programa Excel y las variables fueron medidas con la Escala de Estatones. Así mismo, para determinar la relación entre ambas variables en estudio, se utilizó Chi cuadrado en el software SPSS versión 24.

Para determinar la relación entre ambas variables de estudio, se utilizó la prueba paramétrica coeficiente de Correlación de Pearson.

El Comité de Ética aprobó el estudio, por lo que se solicitó de manera voluntaria la participación de los enfermeros en el estudio mediante el consentimiento informado respetando los principios bioéticos.

\section{RESULTADOS}

En la tabla 1 se observa que el $57,1 \%$ de enfermeros que participaron en el presente estudio oscilan entre las edades de 31 a 35 años, siendo el $62,9 \%$ población femenina y un $81,4 \%$ con tiempo laboral de 3 a 6

Tabla 1. Matriz de datos generales de los enfermeros del Sistema de Atención Móvil de Urgencia (SAMU) Lima - Perú 2017.

\begin{tabular}{lcc}
\hline & \multicolumn{2}{c}{ Enfermeros } \\
\cline { 2 - 3 } Datos generales & $\mathbf{n}$ & \% \\
\hline Edad & 21 & 30,0 \\
$25-30$ & 40 & 57,1 \\
$31-35$ & 07 & 10,0 \\
$36-40$ & 02 & 2,9 \\
41 a más & & \\
Género & 44 & 62,9 \\
Femenino & 26 & 37,1 \\
Masculino & & \\
Tiempo laboral & 0 & 0 \\
$<1$ año & 13 & 18,6 \\
1 - 3 años & 57 & 81,4 \\
3 - 6 años & & \\
Condición laboral & 36 & 51,4 \\
Nombrado & 34 & 48,6 \\
Cas &
\end{tabular}


Tabla 2. Tabla de contingencia para Síndrome de Burnout y Satisfacción Laboral

\begin{tabular}{lcccccc}
\hline & \multicolumn{5}{c}{ Satisfacción Laboral } \\
\cline { 2 - 7 } & \multicolumn{2}{c}{ Insatisfacción } & $\begin{array}{c}\text { Medianamente } \\
\text { Satisfecho }\end{array}$ & \multicolumn{2}{c}{ Satisfecho } \\
\hline $\begin{array}{l}\text { Síndrome de } \\
\text { Burnout }\end{array}$ & $\mathbf{n}$ & $\mathbf{\%}$ & $\mathbf{n}$ & $\mathbf{\%}$ & $\mathbf{n}$ & $\%$ \\
Bajo & 5 & 33,3 & 5 & 33,3 & 5 & 33,3 \\
Medio & 8 & 20,0 & 18 & 45,0 & 14 & 35,0 \\
Alto & 6 & 40,0 & 4 & 26,7 & 5 & 33,3 \\
\hline $\mathrm{p}=2,9$ & & & & & &
\end{tabular}

Tabla 3. Nivel de satisfacción laboral según factores extrínsecos e intrínsecos de los enfermeros del Sistema de Atención Móvil de Urgencia, Lima - Perú 2017.

\begin{tabular}{lcccc}
\hline & \multicolumn{2}{c}{$\begin{array}{c}\text { Factores } \\
\text { Intrínsecos }\end{array}$} & \multicolumn{2}{c}{$\begin{array}{c}\text { Factores } \\
\text { Extrínsecos }\end{array}$} \\
\cline { 2 - 5 } Satisfacción Laboral & $\mathbf{n}$ & $\mathbf{\%}$ & $\mathbf{n}$ & $\mathbf{\%}$ \\
\hline Satisfecho & 22 & 31,4 & 21 & 30,0 \\
Medianamente Satisfecho & 28 & 40,0 & 21 & 30,0 \\
Insatisfecho & 20 & 28,6 & 28 & 40,0 \\
\hline
\end{tabular}

años. En cuanto a la condición laboral, el 51,4\% tenía nombramiento.

En la tabla 2 se observa que no existe relación entre las variables en estudio $(p \geq 0,05)$.

En la Tabla 3 se observa que el $40 \%$ de los enfermeros se encuentran medianamente satisfechos respecto a los factores intrínsecos y el mismo porcentaje presenta insatisfacción en los factores extrínsecos.

En la tabla 4 se observa que del total de enfermeros encuestados, sobre el nivel de satisfacción laboral según los factores extrínsecos la mayoría se encuentran medianamente satisfechos respecto a la relación con los compañeros $(71,4 \%)$, seguido de la interacción con la autoridad $(68,6 \%)$; en el salario predomina una mediana satisfacción $(65,7 \%)$; asimismo se observa igual porcentaje $(44,3 \%)$ de mediana satisfacción e insatisfacción en el horario laboral; también se obtuvo insatisfacción laboral con la mínima diferencia de porcentaje entre beneficios laborales y las normas políticas respectivamente. Respecto a los factores intrínsecos se observa que en los enfermeros del estudio predomina la mediana satisfacción respecto a las dimensiones de oportunidades de aprendizaje (64\%) seguido de logros profesionales (60\%); por el contrario se obtuvo igual porcentaje de satisfacción e insatisfacción laboral en la dimensión de méritos y reconocimiento (34\%), finalizando en insatisfacción respecto a la realización personal $(41,4 \%)$.

En la tabla 5 se muestra las dimensiones de la variable de Síndrome de Burnout, siendo el agotamiento emocional $(42,9 \%)$ de enfermeros que se encuentran con un nivel alto y un menor porcentaje $(24,3 \%)$ en nivel medio; seguido de la dimensión despersonalización $(41,4 \%)$ con nivel bajo pero un valor considerable de enfermeros con nivel alto de la misma, y por último se obtuvo un nivel medio de falta de realización personal $(38,6 \%)$.

\section{DISCUSIÓN}

Según los resultados se identificó que no existe relación entre la variable Satisfacción Laboral y Síndrome de Burnout, coincidiendo con Hernández, Jiménez, Llergo y Perandrés, quienes mencionan que la discrepancia entre las variables probablemente se deba a la realidad socio-económica, atribuyendo que en países con un grado de bienestar inferior, las condiciones laborales/personales más duras y difíciles, son apreciadas como menos estresantes (16). Pero no coinciden con los resultados obtenidos por Solorzano, Rivera, Callan y Moquillaza, quienes mencionan que 
Tabla 4. Satisfacción laboral según factores extrínsecos e intrínsecos de los enfermeros del Sistema de Atención Móvil de Urgencia, Lima - Perú 2017.

\begin{tabular}{lcccccc}
\hline & \multicolumn{3}{c}{ Satisfecho } & \multicolumn{2}{c}{$\begin{array}{c}\text { Medianamente } \\
\text { Insatisfecho }\end{array}$} & \multicolumn{2}{c}{ Insatisfecho } \\
\cline { 2 - 8 } Factores & $\mathbf{n}$ & $\mathbf{\%}$ & $\mathbf{n}$ & $\mathbf{\%}$ & $\mathbf{n}$ & $\mathbf{\%}$ \\
\hline Factores Extrínsecos & & & & & & \\
Relación con los compañeros & 5 & 7,2 & 50 & 71,4 & 15 & 21,4 \\
Beneficios Laborales & 13 & 18,6 & 26 & 37,1 & 31 & 44,3 \\
Interacción con la autoridad & 1 & 1,4 & 48 & 68,6 & 21 & 30,0 \\
Horario Laboral & 8 & 11,4 & 31 & 44,3 & 31 & 44,3 \\
Salario & 3 & 4,3 & 46 & 65,7 & 21 & 30,0 \\
Normas políticas & 16 & 22,9 & 23 & 32,9 & 31 & 44,2 \\
Condiciones físicas & 24 & 34,3 & 30 & 42,9 & 16 & 22,8 \\
Factores Intrínsecos & & & & & & \\
Logros profesionales & 24 & 34,3 & 42 & 60,0 & 4 & 5,7 \\
Realización personal & 19 & 27,2 & 22 & 31,4 & 29 & 41,4 \\
Méritos, reconocimiento & 24 & 34,0 & 22 & 31,0 & 24 & 34,0 \\
Oportunidades de aprendizaje & 9 & 13,0 & 45 & 64,0 & 16 & 23,0 \\
\hline
\end{tabular}

Tabla 5. Nivel de Síndrome de Burnout según dimensiones de los enfermeros del Sistema de Atención Móvil de Urgencia Lima - Perú 2017.

\begin{tabular}{lccccccc}
\hline & \multicolumn{3}{c}{ Nivel de Síndrome de Burnout } \\
\cline { 2 - 7 } Dimensión Síndrome de Burnout & \multicolumn{2}{c}{ Bajo } & \multicolumn{2}{c}{ Medio } & \multicolumn{2}{c}{ Alto } \\
\hline Falta de realización personal & n & \% & n & \% & n & \% \\
Despersonalización & 22 & 31,4 & 27 & 38,6 & 21 & 30,0 \\
Agotamiento emocional & 29 & 41,4 & 18 & 25,7 & 23 & 32,9 \\
\hline
\end{tabular}

existe relación estadísticamente significativa entre las mencionadas variables, por lo que precisan que el agotamiento emocional y la despersonalización se correlacionan indirectamente con la satisfacción laboral, y de forma directa con la realización personal (17).

En cuanto a la Satisfacción laboral según los factores intrínsecos, se obtuvo que un porcentaje importante de los enfermeros se encuentran medianamente satisfechos en lo referente a oportunidades de aprendizaje y logros profesionales, en cuanto a realización personal la mayoría de enfermeros se encuentran insatisfechos, al igual que en méritos, reconocimiento donde los resultados evidencian similitudes porcentuales. Resultados que discrepan con los de Delgado (18), quien menciona que la realización personal en la mayoría de los enfermeros es de medianamente satisfechos y que probablemente se deba a que disfrutan de sus actividades labores y que por lo tanto se sienten bien en su trabajo.
Asimismo, los hallazgos están en desacuerdo con las conclusiones encontradas en el estudio realizado por Castro, quien manifiesta que la mayoría de enfermeros mostraron satisfacción respecto al trabajo en sí y a los logros profesionales, lo cual genera incremento en el compromiso con la institución y fortalece el vínculo laboral; también se enfatiza que probablemente se deba a que se encuentran a gusto con el trabajo que realizan, vocación de servicio, oportunidades de capacitación, adquisición de nuevas experiencias entre otros, los cuales parecen ser predictores favorables para mantenerse motivados (19). Según lo expuesto por los diversos autores y observando la insatisfacción laboral en la dimensión realización personal de los enfermeros del presente estudio, esto probablemente se deba a que se encuentran desanimados con la labor que realizan, desmotivados a seguir con sus objetivos de aprendizaje y por lo tanto a seguir con una rutina laboral.

Siguiendo con dicha variable, en los factores extrínsecos se identificó que un porcentaje importante 
de los enfermeros se encuentran medianamente satisfechos en relación con los compañeros de trabajo, interacción con la autoridad y salario; insatisfechos en cuanto a los beneficios laborales y normas políticas, similares porcentajes de medianamente satisfechos e insatisfechos. Estos resultados son afines con la evidencia en el estudio realizado por Mancilla y Olarte donde se obtuvo que la mayoría del personal de enfermería se sintieron insatisfechos con su labor realizada afectándose en los factores de sistema de trabajo (20). También coincide con los resultados de Morales, que fue calificada como baja en el factor beneficios sociales y/o remuneración alegando a que la institución no se preocupa por las necesidades primordiales del personal y la distribución equitativa a las capacitaciones institucionales (21). Por lo tanto, se podría mencionar que posiblemente la insatisfacción laboral en el estudio según los factores extrínsecos se deba a que el profesional de Enfermería talvez desconoce las normas políticas e institucionales, sumado a que exista indiferencia dentro del ambiente laboral y/o no participa en las actividades sociales dentro de la institución, ocasionando un bajo compromiso institucional laboral, altas inasistencias, bajo clima laboral, que podría afectar al bienestar biopsicosocial del enfermero.

En relación a la variable Síndrome de Burnout y siguiendo la teoría de Maslach en el presente estudio se identificó que en cuanto a la dimensión agotamiento emocional un porcentaje importante de enfermeros se encuentran con nivel alto en lo referente a fatiga (mental, física y emocional). Los resultados mencionados armonizan con el estudio realizado por Jurado, quien concluye que la mayoría de enfermeros presentan un nivel alto, refiriendo a que se sienten cansadas (os) cuando se levantan por las mañanas, expresan que se sienten emocionalmente agotados por el trabajo, creen que están trabajando demasiado, y trabajar todo el día con pacientes implica un gran esfuerzo de su parte y trabajar directamente con personas le produce estrés (22).

Según la dimensión falta de realización personal los enfermeros del presente estudio se encuentran en nivel medio caracterizado por actitudes positivas, entusiasmo, relación personal óptima y gratificación laboral óptima. Resultados que coinciden con el estudio realizado por Castillo, concluyendo que la mayoría del personal de enfermería presenta un nivel medio en dicha dimensión; esto indica que la muestra no se considera del todo realizado a nivel personal y laboral (23).
En la dimensión despersonalización se identificó un nivel bajo de síndrome de burnout. Resultados similares a los encontrados por Oros que menciona que la mayoría de enfermeros presenta nivel bajo, dicho autor menciona que no cree que traten a sus pacientes como objetos. (24).

Según Nieto, Nieto y Jiménez, refieren que en los profesionales de la enfermería en el pre hospitalario suele producirse fatiga física y emocional debido al trabajo agotador y estrés, pudiendo llegar incluso a desarrollar problemas conductuales y padecer el síndrome de burnout, afectando al bienestar y calidad de vida de los trabajadores que lo padecen, viéndose afectado la salud física y mental del enfermero, como también el desarrollo adecuado dentro de la institución y usuarios (25).

La limitación del estudio es que los resultados obtenidos no pueden ser comparados con investigaciones hospitalarias, debido a que son características directas a la entidad, sumado que los resultados son de un profesional que labora en el área pre hospitalaria. Se recomienda realizar mayor estudio con respecto a las dimensiones de la Satisfacción Laboral y Síndrome de Burnout en este tipo de población porque es éste personal quien brinda la primera atención de salud a la población.

\section{CONCLUSIONES}

Según los factores intrínsecos la mayoría se encuentran medianamente satisfechos en relación a las oportunidades de aprendizaje y logros profesionales e insatisfechos en realización personal, similitud porcentual de satisfacción e insatisfacción en cuanto a méritos y reconocimiento. Según los factores extrínsecos la mayoría se encuentra medianamente satisfechos respecto a la relación con sus compañeros, interacción con la autoridad, salario y condiciones físicas; insatisfechas con los beneficios laborales y las normas políticas de la institución, similitud porcentual de medianamente satisfecho e insatisfecho en cuanto a horario laboral.

Respecto a Síndrome de Burnout según la dimensión agotamiento emocional la mayoría se encuentra con nivel alto, nivel bajo en la dimensión despersonalización y nivel medio en la dimensión realización personal. Sin embargo, no existe correlación entre las variables Satisfacción Laboral y Síndrome de Burnout. 


\section{Correspondencia:}

Verónica Gabriel Aranda

Correo electrónico: verito771912@hotmail.com

\section{REFERENCIAS BIBLIOGRÁFICAS}

1. Arias W, Arias G. Relación entre el clima organizacional y la satisfacción laboral en una pequeña empresa del sector privado. Ciencia \& trabajo. 2014;16(51): 185-191.

2. Vaca C, Vaca L, Quintero J.. El clima organizacional y la satisfacción laboral: un análisis cuantitativo riguroso de su relación. AD-minister. 2015;26: 5-15.

3. Pérez-Ciordia I, Guillén-Grima F, Brugos A, Aguinaga I. Satisfacción laboral y factores de mejora en profesionales de atención primaria. In Anales del sistema sanitario de Navarra. 2013;36(2): 253-262.

4. Román A, Matzumura P, Gutiérrez H. (2015). Satisfacción laboral en el Servicio de Rayos X de una Institución Privada de Salud de Lima, Nivel III-4, 2015. Horizonte Médico. 2015; 15(3): 20-25.

5. Menéndez F. Formación superior en prevención de riesgos laborales. Valladolid. España: Ed. LEX NOVA; 2007.

6. Felipe L, Gonzales S. Burnout: consecuencias y soluciones. México: El manual moderno; 2015.

7. Benenson P. El cuidado de los derechos humanos: oportunidades y desafíos para el personal de enfermería y partería. España: Amnistía internacional (EDAI); 2006

8. Vásquez-Manrique J, Maruy-Saito A, Verne-Martin E. Frecuencia del síndrome de burnout y niveles de sus dimensiones en el personal de salud del servicio de emergencia de pediatría del Hospital Nacional Cayetano Heredia en el año 2014: Lima, Perú. Revista de Neuro-Psiquiatría. 2014; 77(3): 168-174.

9. Aldrete M, Navarro C, González R, Contreras M, Pérez J. (2015). Factores psicosociales y síndrome de burnout en personal de enfermería de una unidad de tercer nivel de atención a la salud. Ciencia \& trabajo. 2015; 17(52): 32-36.

10. Luna-Porta L, Mayor-Vega A, Taype-Rondán Á. Síndrome de burnout en estudiantes de pregrado de medicina humana: un problema escasamente estudiado en el Perú. Anales de la Facultad de Medicina. 2015; 76(1): 83-84.

11. Montoya P, Beiio-Escamlila N, Bermúdez N, Burgos F, Fuentealba M, Padilla A. Satisfacción Laboral y su relación con el clima organizacional en funcionarios de una universidad estatal chilena. Ciencia $\&$ trabajo. 2017; 19(58): 7-13.

12. Malander M. Síndrome de Burnout y Satisfacción Laboral en Docentes de Nivel Secundario. Ciencia \& trabajo. 2016; 18(57): 177-182.
13. Maticorena-Quevedo J, Beas R, Anduaga-Beramendi A, Mayta-Tristán P. Prevalencia del síndrome de burnout en médicos y enfermeras del Perú. Revista Peruana de Medicina Experimental y Salud Pública. 2016; 33 (2): 241-247.

14. Román A, Matzumura J, Gutiérrez H. Satisfacción laboral en el Servicio de Rayos X de una Institución Privada de Salud de Lima, Nivel III-4, 2015. Horizonte Médico. 2015; 15(3): 20-25.

15. Llaneza J. (2008). Ergonomía y psicosociología aplicada. Ed. 10. España: LEX NOVA; 2015.

16. Hernández M, Jiménez A, Llergo M, Mazzoni L, Perandrés A. Síndrome de burnout y safisfacción laboral en el personal sanitario de España y Argentina. Reidocrea. 2014; 3: 60-68.

17. Solórzano M, Rivera-Felix M, Callan-Zavaleta R, Moquillaza-Alcántara V. (2016). Relación entre las dimensiones del Síndrome de Burnout y la satisfacción laboral en obstetras de un hospital del cono norte de Lima. Revista Peruana de Obstetricia y Enfermería. 2016; 12(2):0-0.

18. Delgado S. Nivel de satisfacción laboral del personal de enfermería en centro quirúrgico en la Clínica Centenario Peruano Japonés 2016. Lima, Perú: Universidad Nacional de San Marcos; 2017.

19. Castro C. Satisfacción laboral de los profesionales de enfermería del Hospital Pablo Arturo Suárez, periodo agosto - noviembre 2013. Quito, Ecuador: Pontificia Universidad Católica del Ecuador; 2014.

20. Mancilla L, Olarte L. Satisfaccion laboral y estrés ocupacional en el personal de enfermería del servicio de emergencia y medicina del Hospital departamental de Huancavelica Zacarías Correa Valdivia. Huancavelica: Universidad Nacional de Huancavelica; 2015.

21. Morales E. Nivel de satisfacción laboral del profesional de enfermería en el servicio de centro quirúrgico Hospital Nacional Alberto Sabogal Sologuren. Lima, Perú: Universidad Nacional de San Marcos; 2016.

22. Jurado V. Síndrome de Burnout en el profesional de enfermería que trabaja en el Hospital Santa Rosa de Puerto Maldonado. Puerto Maldonado, Perú: Universidad Nacional Amazónica Madre de Dios; 2016.

23. Castillo S. Nivel de síndrome de burnout (desgaste laboral) en el personal de Enfermería del Hospital Regional de Huehuetenango Dr. Jorge Vides Molina. Guatemala: Universidad Rafael Landívar; 2016.

24. Oros D. Nivel del Síndrome de Burnout en el personal de enfermería de la UCIP, del Hospital Nacional A. Sabogal S. Lima, Perú: Universidad Nacional San Marcos; 2016.

25. Nieto L, Nieto C, Jiménez M. Los efectos del trabajo en personal sanitario. Madrid. España: Ed. Dykinson Ebook; 2015. 